\title{
Expression of the flp proteins by Haemophilus ducreyi is necessary for virulence in human volunteers
}

\author{
Diane M Janowicz ${ }^{1 *}$, Sean A Cooney ${ }^{1}$, Jessica Walsh² ${ }^{2}$ Beth Baker ${ }^{3}$, Barry P Katz ${ }^{1}$, Kate R Fortney ${ }^{1}$, Beth W Zwickl', \\ Sheila Ellinger ${ }^{1}$ and Robert S Munson $\mathrm{Jr}^{3,4}$
}

\begin{abstract}
Background: Haemophilus ducreyi, the causative agent of the sexually transmitted disease chancroid, contains a flp (fimbria like protein) operon that encodes proteins predicted to contribute to adherence and pathogenesis. $H$. ducreyi mutants that lack expression of Flp1 and Flp2 or TadA, which has homology to NTPases of type IV secretion systems, have decreased abilities to attach to and form microcolonies on human foreskin fibroblasts (HFF). A tadA mutant is attenuated in its ability to cause disease in human volunteers and in the temperature dependent rabbit model, but a flp 1 flp2 mutant is virulent in rabbits. Whether a flp deletion mutant would cause disease in humans is not clear.

Results: We constructed 35000HPAflp1-3, a deletion mutant that lacks expression of all three Flp proteins but has an intact tad secretion system. 35000HPAflp 1-3 was impaired in its ability to form microcolonies and to attach to HFF in vitro when compared to its parent (35000HP). Complementation of the mutant with flp 1-3 in trans restored the parental phenotype. To test whether expression of Flp1-3 was necessary for virulence in humans, ten healthy adult volunteers were experimentally infected with a fixed dose of $35000 \mathrm{HP}$ (ranging from 54 to 67 CFU) on one arm and three doses of 35000HPAflp1-3 (ranging from 63 to 961 CFU) on the other arm. The overall papule formation rate for the parent was 80\% (95\% confidence interval, $\mathrm{Cl}, 55.2 \%-99.9 \%$ ) and for the mutant was $70.0 \%$ (95\% Cl, 50.5\%-89.5\%) ( $P=0.52$ ). Mutant papules were significantly smaller (mean, $11.2 \mathrm{~mm}^{2}$ ) than were parent papules $\left(21.8 \mathrm{~mm}^{2}\right) 24 \mathrm{~h}$ after inoculation $(P=0.018)$. The overall pustule formation rates were $46.7 \%(95 \% \mathrm{Cl} 23.7$ $69.7 \%)$ at 30 parent sites and $6.7 \%(95 \% \mathrm{Cl}, 0.1-19.1 \%)$ at 30 mutant sites $(P=0.001)$.

Conclusion: These data suggest that production and secretion of the Flp proteins contributes to microcolony formation and attachment to HFF cells in vitro. Expression of flp 1-3 is also necessary for $\mathrm{H}$. ducreyi to initiate disease and progress to pustule formation in humans. Future studies will focus on how Flp proteins contribute to microcolony formation and attachment in vivo.
\end{abstract}

\section{Background}

The tad (tight adherence) locus is present in many Gram-positive and Gram-negative bacteria as well as the Archaea and likely represents an ancient subtype of type IV secretion systems that was horizontally transferred to many bacterial species early in the course of evolution. The tad genes are located on a mobile genomic island coined "the widespread colonization island" by Figurski

\footnotetext{
* Correspondence: dmjanowi@iupui.edu

'Department of Medicine, Indiana University, School of Medicine, Indianapolis, IN 46202, USA

Full list of author information is available at the end of the article
}

and coworkers [1]. The functions of the tad locus gene products have been best described for Aggregatibacter (formerly Actinobacillus) actinomycetemcomitans, where they are essential for adherence, biofilm formation, and pathogenesis. The Tad proteins are predicted to form a macromolecular transport system for the assembly and secretion of fimbria or pili, which mediate adherence of the organism to different surfaces [2].

Haemophilus ducreyi is a gram-negative coccobacillus that causes the sexually transmitted genital ulcer disease chancroid, which is a public health concern because it increases the risk of transmission and acquisition of

\section{() Biomed Central}


human immunodeficiency virus-1. $H$. ducreyi contains a $12.8 \mathrm{~kb} f l p$ (fimbria like protein) operon with 15 genes (flp1-flp2-flp3-orfBC-rcpAB-orfD-tadABCDEFG) that encode products with homology to proteins encoded by the tad locus in A. actinomycetemcomitans [3,4]. H. ducreyi mutants that lack expression of either Flp1 and Flp2, which encode fimbria like proteins, or $\operatorname{tad} A$, which has homology to NTPases of type IV secretion systems, have decreased abilities to attach to human foreskin fibroblasts (HFF) and to form microcolonies on HFF $[4,5]$. A mutant deficient in the expression of $\operatorname{tad} A$ was significantly but partially attenuated in the temperature dependent rabbit model of experimental chancroid, while a mutant lacking $f l p 1$ and $f l p 2$ expression was fully virulent in this model [4,5]. Thus, whether FlpTad-mediated adherence and/or microcolony formation are critical factors in the virulence of $H$. ducreyi is unclear [6].

In experimental and natural infection in humans, $H$. ducreyi forms aggregates, the first step in microcolony formation, and colocalizes with polymorphonuclear leukocytes and macrophages, which fail to ingest the organism. In human inoculation experiments, a $\operatorname{tad} A$ mutant is highly attenuated for virulence; whether the observed attenuation is due to the lack of secretion of the Flp proteins or other unidentified effectors by the tad locus is unclear [5]. Given the discrepancy in virulence between the $\operatorname{tad} A$ mutant and the flp1flp 2 mutant in the temperature dependent rabbit model [5], here we constructed and characterized a flp1-3 deletion mutant. We tested the flp1-3 mutant for its ability to cause disease in human volunteers and its ability to form microcolonies and adhere to human fibroblasts. Our data indicate that expression of Flps is required for virulence and that Flp-Tad mediated adherence correlates with the virulence of $H$. ducreyi in humans. To our knowledge, this study is the first to provide definitive proof that expression of the Flp proteins is required for the virulence of a bacterial pathogen in humans.

\section{Results}

\section{Construction and characterization of $35000 \mathrm{HP} \Delta \mathrm{flp} 1-3$}

An unmarked, in frame deletion mutant of the flp1, flp2, and flp3 genes was constructed in $35000 \mathrm{HP}$ using recombineering technology and designated 35000HP $\Delta$ flp1-3 [7,8]. Sequence analysis of $35000 \mathrm{HP} \Delta f l p 1-3$ confirmed that flp1, flp 2 and $f l p 3$ had been replaced by a short ORF that consisted of the upstream region of $f l p 1$, the start codon of $f l p 1,81 \mathrm{bp}$ encoding a scar peptide, and the last 21 bp of $f l p 3$, including its stop codon. By qRT-PCR, the expression levels of $\operatorname{tad} A$ and $t a d G$, two genes downstream of flp3, were similar in $35000 \mathrm{HP} \Delta$ flp1-3 compared to $35000 \mathrm{HP}$ (data not shown), suggesting that the remainder of the flp operon was normally transcribed. $35000 \mathrm{HP}$ and $35000 \mathrm{HP} \Delta$ flp1-3 demonstrated identical growth rates in broth (data not shown). The LOS profiles and OMP patterns as analyzed by SDS-PAGE were similar for the mutant and the parent (data not shown).

\section{Human inoculation experiments}

To determine whether the Flp proteins play a role in pathogenesis, $35000 \mathrm{HP} \Delta f l p 1-3$ was compared with $35000 \mathrm{HP}$ for virulence using a mutant parent comparison trial in the human model of infection. Ten healthy adults (six males, four females; 5 Caucasian, 5 black; age range 32 to 59 ; mean age \pm standard deviation, $48 \pm 9$ years) volunteered for the study. Three subjects (volunteers 333, 334, and 335) were inoculated in the first iteration, three subjects (volunteers 336, 337, and 338) in the second iteration, one subject (volunteer 341) in the third iteration and three subjects (volunteers 342, 343 , and 344) in the fourth iteration. In the first iteration, each subject was infected with a fixed EDD (61 CFU) of $35000 \mathrm{HP}$ at three sites on one arm and varying EDDs $(63,125$, and $249 \mathrm{CFU})$ of $35000 \mathrm{HP} \Delta$ flp $1-3$ on the other arm (Table 1). Since the ability of the mutant to form pustules seemed impaired (Table 1), we increased the dose of the mutant in subsequent iterations, as per protocol. In the second iteration, volunteers were inoculated with $65 \mathrm{CFU}$ of the parent and 66, 131, and 262 CFU of the mutant (Table 1). In the third iteration, the volunteer was infected with $67 \mathrm{CFU}$ of $35000 \mathrm{HP}$ and 240, 480, and $961 \mathrm{CFU}$ of the mutant (Table 1). In the fourth iteration, volunteers were inoculated with 54 CFU of the parent and 104, 208 and 415 CFU of the mutant (Table 1).

The overall papule formation rate for the parent was $80 \%$ (95\% confidence interval, CI, 55.2\%-99.9\%) at 30 sites and for the mutant was $70.0 \%$ (95\% CI, 50.5\%$89.5 \%)$ at 30 sites $(P=0.52)$. Mutant papules were significantly smaller (mean, $11.2 \mathrm{~mm}^{2}$ ) than were parent papules $\left(21.8 \mathrm{~mm}^{2}\right) 24 \mathrm{~h}$ after inoculation $(P=0.018)$. The overall pustule formation rates were $46.7 \%$ (95\% CI 23.7-69.7\%) at 30 parent sites and 6.7\% (95\% CI, 0.1$19.1 \%)$ at 30 mutant sites $(P=0.001)$. Mutant pustules formed at only two sites in one volunteer. These results indicate that expression of one or more of the flp $1, f l p 2$, and flp3 genes in the context of the intact secretion/ assembly complex is necessary for $H$. ducreyi to initiate disease and progress to pustule formation in humans.

$H$. ducreyi was recovered intermittently from surface cultures. Of the 30 sites that were inoculated with the parent, $11(36.7 \%)$ yielded at least one positive surface culture, while 3 of 30 mutant sites $(10.0 \%)$ yielded a positive surface culture $(P=0.019)$. All colonies recovered from sites inoculated with the parent $(n=626)$ or the mutant $(\mathrm{n}=39)$ and colonies from the parent $(\mathrm{n}=$ 
Table 1 Response to inoculation of live H.ducreyi strains

\begin{tabular}{|c|c|c|c|c|c|c|}
\hline Volunteer & Gender $^{a}$ & $\begin{array}{c}\text { Days of } \\
\text { Observation }\end{array}$ & Isolate $^{b}$ & Dose (cfu) & $\begin{array}{c}\text { No. of } \\
\text { Initial } \\
\text { Papules }\end{array}$ & $\begin{array}{c}\text { No. of } \\
\text { Pustules }\end{array}$ \\
\hline \multirow[t]{2}{*}{333} & $F$ & 14 & $P$ & 61 & 3 & 1 \\
\hline & & & M & 63-249 & 2 & 0 \\
\hline \multirow[t]{2}{*}{334} & $M$ & 9 & $P$ & 61 & 3 & 0 \\
\hline & & & M & $63-249$ & 2 & 0 \\
\hline \multirow[t]{2}{*}{335} & $M$ & 7 & $P$ & 61 & 3 & 1 \\
\hline & & & M & $63-249$ & 3 & 0 \\
\hline \multirow[t]{2}{*}{336} & $\mathrm{~F}$ & 6 & $P$ & 65 & 0 & 0 \\
\hline & & & M & $66-261$ & 3 & 0 \\
\hline \multirow[t]{2}{*}{337} & $M$ & 7 & $P$ & 65 & 0 & 0 \\
\hline & & & M & $66-261$ & 1 & 0 \\
\hline \multirow[t]{2}{*}{338} & $\mathrm{~F}$ & 8 & $P$ & 65 & 3 & 2 \\
\hline & & & M & $66-261$ & 0 & 0 \\
\hline \multirow[t]{2}{*}{341} & M & 8 & $P$ & 67 & 3 & 2 \\
\hline & & & $M$ & $240-961$ & 3 & $2^{c}$ \\
\hline \multirow[t]{2}{*}{342} & $\mathrm{~F}$ & 7 & $P$ & 54 & 3 & 3 \\
\hline & & & M & $104-415$ & 3 & 0 \\
\hline \multirow[t]{2}{*}{343} & M & 6 & $P$ & 54 & 3 & 3 \\
\hline & & & M & $104-415$ & 2 & 0 \\
\hline \multirow[t]{2}{*}{344} & M & 7 & $P$ & 54 & 3 & 2 \\
\hline & & & M & $104-415$ & 2 & 0 \\
\hline
\end{tabular}

142) and mutant $(\mathrm{n}=143)$ inocula were tested for the presence of $f l p 1-f l p 2-f l p 3$ and $f g b A$ sequences by colony hybridization. The $f g b A$ probe hybridized to all the colonies, while the flp1-2-3 probe hybridized only to the colonies obtained from the parent inoculated sites or the parent inocula. Thus, there was no cross contamination of mutant and parent sites during the course of the trial.

\section{Deletion of flp1flp2flp3 affects attachment of 35000HP to HFF cells and microcolony formation}

To confirm the phenotype of $35000 \mathrm{HP} \Delta f l p 1-3$, we complemented $35000 \mathrm{HP} \Delta$ flp1-3 in trans by electroporating it with plasmid pJW1, which encodes the Flp1, Flp2 and Flp3. flp1, flp2, and $f l p 3$ encode proteins with predicted molecular weights of $9.3 \mathrm{kDa}, 8.9 \mathrm{kDa}$, and $9.9 \mathrm{kDa}$, respectively. Western blot analysis with a polyclonal sera that binds to Flp1 and Flp2 confirmed that $35000 \mathrm{HP} \Delta f l p 1-3$ (pLSSK) lacked the ability to express the Flp1 and Flp2 proteins (Figure 1, lane 2) compared to $35000 \mathrm{HP}$ (pLSSK) (Figure 1, lane 1). Complementation of $35000 \mathrm{HP} \Delta f l p 1-3$ with plasmid pJW1 resulted in restoration of the expression of the Flp1 and Flp2 proteins as determined by Western blot (Figure 1, lane 3 ).

$35000 \mathrm{HP}(\mathrm{pLSSK}), 35000 \mathrm{HP} \Delta$ flp $1-3$ (pLSSK), and $35000 \mathrm{HP} \Delta f l p 1-3(\mathrm{pJW} 1)$ were also tested for their abilities to bind confluent HFF monolayers. 35000HP $\Delta$ flp 1-3 (pLSSK) significantly attached to HFF cells at lower levels (geomean \pm standard deviation, $26.0 \% \pm 15.0 \%)$ than did $35000 \mathrm{HP}(\mathrm{pLSSK})(100 \% \pm 29.0 \%)(P=0.018)$ (Figure 2$)$. $35000 \mathrm{HP} \Delta$ flp1-3(pJW1) adhered to HFF cells $(92.0 \% \pm$ $18.0 \%)$ at significantly higher levels than $35000 \mathrm{HP} \Delta$ flp1-3 (pLSSK) $(P=0.010)$ and at similar levels as $35000 \mathrm{HP}$ (pLSSK) $(P=0.32)$ (Figure 2$)$.

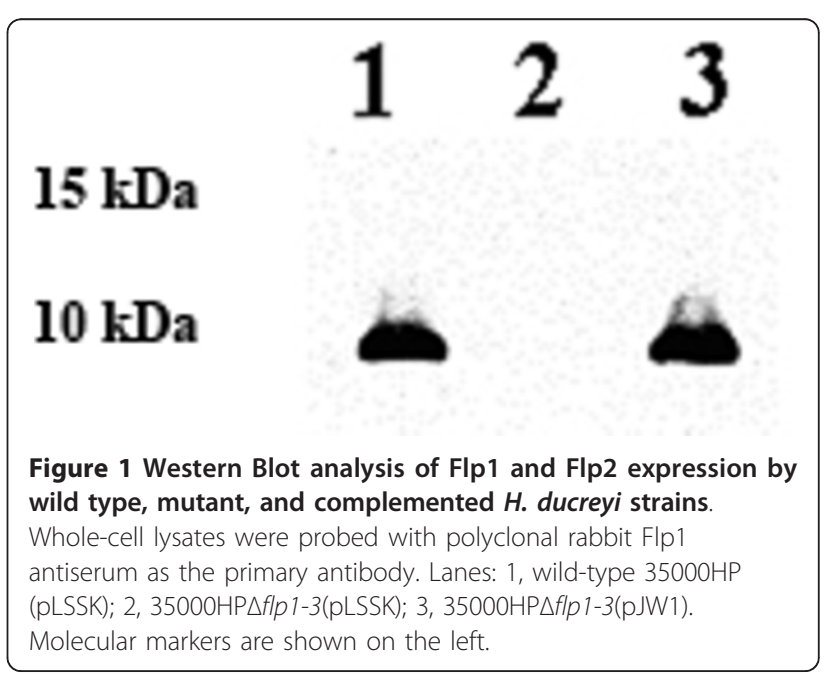




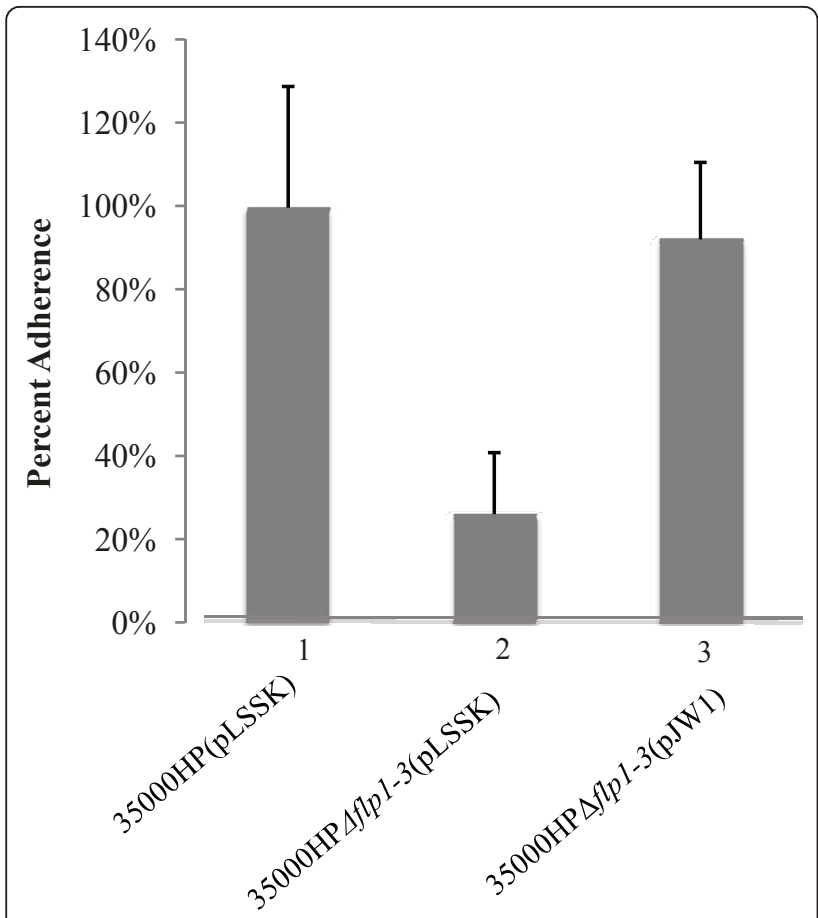

Figure 2 Quantitative measurement of the binding of wild type, mutant, and complemented $\mathrm{H}$. ducreyi strains to HFF cells. Assays were performed as described in Materials and Methods. The data represented are a composite of five separate experiments. Bars: 1, wild-type $35000 \mathrm{HP}(\mathrm{pLSSK}) ; 2,35000 \mathrm{HP} \triangle \mathrm{flp} 1-3$ (pLSSK); 3, 35000HPAflp1-3(pJW1).

$35000 \mathrm{HP}$ (pLSSK), 35000HP $\Delta$ flp1-3(pLSSK), and $35000 \mathrm{HP} \Delta f l p 1-3$ (pJW1) were also compared for their abilities to form microcolonies after $24 \mathrm{~h}$ incubation with confluent HFF monolayers. 35000HP formed numerous, densely populated microcolonies on the surfaces of HFF cells [4] (Figure 3A). 35000HP $\Delta$ flp1-3 (pLSSK) formed sparse and very small microcolonies (Figure 3B) when compared to 35000HP; the complemented mutant demonstrated a restored phenotype similar to $35000 \mathrm{HP}(\mathrm{pLSSK})$ (Figure 3C). Thus, complementation of the mutant restored the parental phenotypes.

\section{Discussion}

For this study, we focused on whether the expression of the Flp proteins was necessary for virulence of $H$. ducreyi. We constructed an unmarked, in frame deletion mutant lacking the flp 1 flp 2 flp 3 genes in $35000 \mathrm{HP}$ using a recombineering strategy $[8,9]$ and found that $35000 \mathrm{HP}$ flp 1-3 was significantly impaired in its ability to cause disease in the human model of infection. $f l p 1-3$ joins $h g b A, d s r A, n c a A, l s p A 1-l s p A 2, p a l, \operatorname{tad} A \operatorname{sap} B C$ and $c p x A$ as the ninth gene required for full virulence by $H$. ducreyi in the human inoculation experiments $[9,10]$ (unpublished observations).

The flp-tad gene cluster is constitutively transcribed as a single polycistronic operon in vitro [4]. Relative to its expression during in vitro growth, $t a d A$ transcripts are enriched in experimental pustules, suggesting that the flp-tad operon is upregulated in vivo [11]. CpxRA is the only obvious intact two-component signal transduction system contained in $H$. ducreyi. Transcription of $f l p 1-3$ and several other major virulence determinants are negatively regulated by conditions that favor phosphorylation of CpxR $[9,12,13]$. Purified recombinant CpxR interacts with the promoter regions of the $f l p$ operon in electrophoretic mobility shift assays [13]. Deletion of cpxA leads to loss of CpxA phosphatase activity, activates $C p x R$, and cripples the ability of $H$. ducreyi to infect humans [9]. In contrast, a $c p x R$ deletion mutant has no effect on or upregulates the expression of virulence determinants and is fully virulent in human volunteers [13]. Taken together, the data suggest that the flptad operon may be upregulated in vivo due to downregulation of CpxRA.

The human inoculation experiments are limited in that we are precluded by several regulatory bodies from

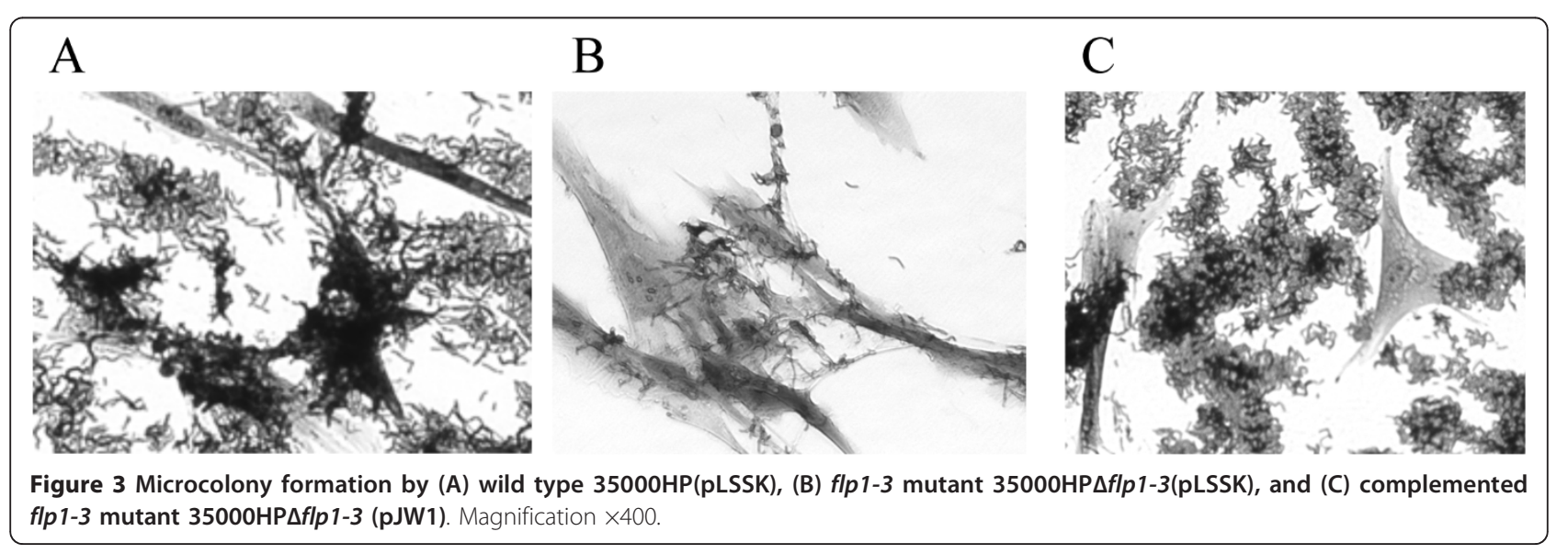


testing trans-complemented mutants in humans. However, complementation of $35000 \mathrm{HP} \Delta f l p 1-3$ in trans restored the ability of the mutant to form microcolonies and bind to HFF cells, suggesting that the phenotype of the mutant is due to the deletion of the $f l p$ genes. In the human inoculation experiments, we use $35000 \mathrm{HP}$ to examine the role of virulence factors in $H$. ducreyi pathogenesis. There are two classes of $H$. ducreyi strains, which express different immunotypes and proteomes $[14,15]$. Although we were able to amplify flp1-3 alleles from six class I and three class II strains (data not shown), attempts to sequence the amplicons were unsuccessful, so we do not know if there is a difference in the $f l p$ genes in the class I and class II strains. $35000 \mathrm{HP}$ is a class I strain; whether the Flp proteins play a role in the virulence of class II strains is unknown.

We previously reported that a $\operatorname{tad} A$ mutant is attenuated for pustule formation in the human challenge model [5]. However, the $\operatorname{tadA}$ mutant, but not a flp 1 flp2 double mutant, is attenuated in the rabbit model of chancroid $[4,5]$. Nika et al previously reported that both the flp1flp2 mutant and the $\operatorname{tad} A$ mutant demonstrate decreased abilities to attach to HFF cells and form fewer microcolonies on HFF cells [4]. These data suggested that microcolony formation by itself is not a virulence factor for $H$. ducreyi. Although $H$. ducreyi does not appear to co-localize with fibroblasts in experimental or natural chancroid $[16,17]$, our data indicate that adherence to HFF cells in vitro correlates with the virulence of $H$. ducreyi in humans. Similarly, both $f l p 1$ and $\operatorname{tad} A$ mutants fail to colonize or cause disease in a rat infection model with $A$. actinomycetemcomitans [1], and both flp1 mutant and tadD mutants of Pasteurella multocida are attenuated in the septicemic mouse model $[18,19]$.

In $A$. actinomycetemcomitans, Flp pili are assembled as bundles of long fibers in which Flp1 is the major structural component $[3,20]$. However, there is no evidence that the Flp proteins are assembled into a pilus-like structure in $H$. ducreyi [4]. Several bacterial species including $A$. actinomycetemcomitans have two flp genes [2]. H. ducreyi contains three flp genes, which have between $50-80 \%$ similarity to one another [4]. Deletion of $f l p 1$ and $f l p 2$ results in decreased adherence of $H$. ducreyi to HFF cells and subsequent microcolony formation [4]; the function of Flp3 is unclear.

In vitro, $H$. ducreyi forms microcolonies, a key step in biofilm formation. In vivo, $H$. ducreyi forms aggregates and colocalizes with macrophages, PMNs, collagen and fibrin $[16,17]$. H. ducreyi contains a $l u x S$ homologue that has autoinducer (AI-2) activity in a Vibrio harveyibased reporter system, and a $\operatorname{luxS}$ mutant is partially attenuated for virulence in human volunteers [21].
Taken together, these data suggest that the formation of microcolonies, aggregates and quorum sensing mechanisms may be important for $H$. ducreyi pathogenesis. Whether the Flp proteins contribute to this process by mediating attachment to host cells or initiating microcolony formation in the skin remains a subject for future investigation.

\section{Conclusions}

We have constructed an unmarked, in frame deletion mutant lacking the flp 1 flp $2 f l p 3$ genes in $H$. ducreyi strain $35000 \mathrm{HP}$. The deletion mutant, 35000HP $\Delta$ flp1-3, has an intact tad secretion system. Our data show that production and secretion of the Flp proteins contributes to microcolony formation and attachment of $35000 \mathrm{HP}$ to HFF cells in vitro. Complementation of the mutant with $f l p 1-3$ in trans restored the parental phenotype. Additionally, expression of Flp1-3 is necessary for $H$. ducreyi to initiate disease and progress to pustule formation in humans. Future studies will focus on how Flp proteins contribute to microcolony formation and attachment in vivo.

\section{Methods}

\section{Bacteria and culture conditions}

$35000 \mathrm{HP}$ is a human-passaged (HP) variant of strain 35000 and has been reported previously [22]. H. ducreyi strains were grown on chocolate agar plates supplemented with $1 \%$ IsoVitaleX at $33^{\circ} \mathrm{C}$ in $5 \% \mathrm{CO}_{2}$. For the human inoculation experiments, $H$. ducreyi was grown in a protease peptone broth-based medium supplemented with $50 \mu \mathrm{g}$ of hemin per $\mathrm{ml}, 1 \%$ IsoVitaleX and $5 \%$ heat-inactivated fetal calf serum (FCS) as described [23] or in a Columbia broth based medium with $2.5 \%$ heatinactivated FCS for other experiments. When appropriate, the media were supplemented with chloramphenicol, spectinomycin, or kanamycin at $0.3 \mu \mathrm{g} / \mathrm{ml}, 200 \mu \mathrm{g} /$ $\mathrm{ml}$, or $20 \mu \mathrm{g} / \mathrm{ml}$, respectively, to maintain plasmids or select for chromosomal integration of antibiotic resistance cassettes. E. coli strains were grown in Luria-Bertani broth or plates at $37^{\circ} \mathrm{C}$, except for strain DY380, which was grown or maintained at $32^{\circ} \mathrm{C}$ and induced to express $\lambda$ recombinase at $42^{\circ} \mathrm{C}$.

\section{Construction and characterization of a flp1-3 mutant of strain 35000HP}

An unmarked, in frame deletion mutant of the $f l p 1$, flp2, and flp 3 genes was made in $H$. ducreyi strain $35000 \mathrm{HP}$ using Flippase (FLP) recombinase technology as described previously $[8,9]$. Briefly, two 70 bp primers, P1 and P2, were designed for construction of a cassette (Table 2). The 3 ' end of each of these primers contained 20 bp complementary to regions 5' and 3' of a spectinomycin cassette flanked by FLP Recognition Target (FRT) sites in 
Table 2 Primers used in this study

\begin{tabular}{ll}
\hline Primer & Sequence \\
\hline P1 2 & TAACCTAAAAAAACAACATAATTATTTTATATTTGGAGAAAAAGATATGATTCCGGGGATCCGTCGACC \\
\hline P3 & GTATATATGGCACATATAAATTATGTGTTTATAATCTACCTTATTGAATGTAGGCTGGAGCTGCTTCG \\
\hline P4 P5 & CGGTCACGATGGTTCAATGTCT \\
\hline P6 & AGCGTTGACATCATCACCATACT \\
\hline P7 & CCACTCGAAAGCGAAACTTGT \\
\hline P8 PATCTCGAGCGCCACACTATCCAC \\
\hline P10
\end{tabular}

pRSM2832 [8]. The 5' portion of the P1 and P2 primers were homologous to regions 5' and 3' of $\mathrm{H}$. ducreyi flp1 and flp3, respectively. PCR of pRSM2832 with P1 and P2 yielded a $2 \mathrm{~Kb}$ amplicon that contained the spectinomycin cassette flanked by FRT sites and 50 bp of DNA homologous to regions 5' and 3 ' of $H$. ducreyi flp 1 and $f l p 3$, respectively. This amplicon was electroporated into $E$. coli DY380 harboring a cosmid size pBeloBAC clone containing the $f l p$ operon and flanking DNA. After induction of $\lambda$ recombinase in this strain, spectinomycin-resistant clones were isolated. One clone was further characterized to demonstrate that the $f l p 1,-2$ and -3 genes were replaced with the spectinomycin cassette, with the exception of the flp 1 start codon and the terminal 21 bp of the flp3 ORF. The construct was confirmed by sequence analysis.

The pBeloBAC clone containing the insertion/deletion mutation in the $f l p$ genes was used as a template for PCR. The amplicon containing the insertionally inactivated $f l p 1 f l p 2 f l p 3$ genes and approximately 500 bp of flanking DNA 5' and 3' to the cassette was ligated into the suicide vector, pRSM2072, and then electroporated into $35000 \mathrm{HP}$. Cointegrates were selected by growth on spectinomycin, then resolved by passage on plates containing spectinomycin and 5-bromo-4-chloro-3-indoly$\beta$-D-galactopyranoside (X-Gal) [24]. Allelic exchange was confirmed by colony PCR.

To make an unmarked mutant, the plasmid, pRSM2975, which contains a temperature sensitive replicon, a kanamycin resistance cassette, and FLP recombinase under the control of the tet repressor, was transformed into the mutant [9]. Transformants were selected and maintained at $32^{\circ} \mathrm{C}$ on chocolate agar containing kanamycin. The FLP recombinase was induced to catalyze excision of the spectinomycin cassette resulting in a short unmarked ORF in place of the $f l p 1, f l p 2$ and $f l p 3$ genes and the plasmid was cured as described previously [9]. Colonies were replica plated on chocolate agar containing no antibiotics, $200 \mu \mathrm{g} / \mathrm{ml}$ of spectinomycin, and $20 \mu \mathrm{g} / \mathrm{ml}$ of kanamycin to identify clones that lost both the temperature sensitive plasmid and the spectinomycin cassette. The plasmid and the spectinomycin cassette were lost in 3/120 (2.5\%) of the clones tested. One clone that had a deletion of the expected size by colony PCR was designated $35000 \mathrm{HP} \Delta$ flp1-3.

Lipooligosaccharide (LOS) and outer membrane proteins (OMPs) were prepared from 35000HP and $35000 \mathrm{HP} \Delta f l p 1-3$ and were analyzed by sodium dodecyl sulfate-polyacrylamide gel electrophoresis, as described [25]. The growth of parent and mutant in broth cultures were also compared.

\section{RNA isolation and Real Time PCR}

Bacterial RNA was prepared from mid-log phase organisms by using TRIzol Reagent (Invitrogen) according to manufacturer's instructions. After isolation, RNA was treated twice with DNaseI (Ambion) for 1 hour at $37^{\circ} \mathrm{C}$ and then purified by using the RNeasy system (Qiagen). Samples were checked by Agilent analysis. After optimizing primers so that their efficiencies were greater than $95 \%$, we examined the level of transcript expression in RNA isolated from $35000 \mathrm{HP}$ and $35000 \mathrm{HP} \Delta f l p 1-3$. Using each bacterial RNA, and either the $\operatorname{tad} A$ primers (P3 and P4) (Table 2) or the tadG (P5 and P6) primers (Table 2) and SYBR Green, reactions were performed in triplicate using an ABI PRISM 7000 Sequence Detector (Applied Biosystems). Data were expressed as fold change of $\operatorname{tad} A$ and $\operatorname{tad} G$ in the mutant relative to the parent.

\section{Complementation of 35000HPAflp1-3}

To complement $35000 \mathrm{HP} \Delta f l p 1-3$ in trans, the $f l p 1$, flp 2 and flp3 ORFs were amplified using the P7 primer with a BamH1 linker and the P8 primer with an XhoI linker. The resulting $1.58-\mathrm{kb}$ amplicon was ligated into pCR- 
XL-TOPO (Invitrogen, Calsbad, Calf.). Transformants were selected on Luria-Bertani plates supplemented with kanamycin $(50 \mu \mathrm{g} / \mathrm{ml})$. The $1.58 \mathrm{~kb}$ insert was released from the vector by digestion with BamHI and XhoI, ligated into pLSSK [26], and then transformed into $E$. coli $\mathrm{DH} 5 \alpha$. The plasmid was confirmed by restriction mapping and designated pJW1. $H$. ducreyi $35000 \mathrm{HP} \Delta$ flp1-3 was electroporated with pJW1. As controls, $35000 \mathrm{HP}$ and $35000 \mathrm{HP} \Delta$ flp 1-3 were electroporated with pLSSK. Transformants were selected on chocolate agar plates containing streptomycin $(50 \mu / \mathrm{ml})$ and transformants were saved and designated $35000 \mathrm{HP} \Delta$ flp 1-3 (pJW1), 35000HP(pLSSK) and 35000HPAflp1-3(pLSSK).

\section{SDS-PAGE and Western Blot Analysis}

Whole cell lysates were prepared from $35000 \mathrm{HP} \Delta f \mathrm{fl} 1-3$ (pJW1), 35000HPAflp1-3(pLSSK), and 35000HP(pLSSK) and subjected to SDS-PAGE as previously described [27]. In Western Blot analysis, whole cell lysates were probed with rabbit polyclonal sera that bind to Flp1 and Flp2 (kindly provided by Eric J. Hansen) as described elsewhere [4].

\section{Human inoculation protocol}

Stocks of $35000 \mathrm{HP}$ and $35000 \mathrm{HP} \Delta$ flp1-3 were prepared according to the US Food and Drug Administration guidelines (BB-IND 13046). For the human inoculation protocol, healthy adult male and female volunteers over 21 years of age were recruited for the study. Subjects gave informed consent for participation and for human immunodeficiency virus (HIV) serology, in accordance with the human experimentation guidelines of the U.S. Department of Health and Human Services and the institutional ethics committee of Indiana University-Purdue University of Indianapolis. The experimental protocol, preparation and inoculation of the bacteria, calculation of the estimated delivered dose (EDD), and clinical observations were all done exactly as described previously $[10,28]$. Subjects were observed until they reached clinical endpoint, which was defined as resolution of all sites, development of a pustule that was either painful or $>6 \mathrm{~mm}$ in diameter, or 14 days after inoculation. Subjects were then treated with one dose of oral ciprofloxacin as described [29]. Comparison of papule and pustule formation rates for the two strains were performed using a logistic regression model with generalized estimating equations (GEE) to account for the correlation among sites within the same individual, as previously described [28]. The GEE sandwich estimate for the standard errors was used to calculate $95 \%$ confidence intervals $(95 \% \mathrm{CI})$ for these rates.

To confirm that the strains contained or lacked the flp1flp 2 flp 3 genes, colonies from the inocula, surface cultures and biopsy specimens were replica plated and grown on nitrocellulose filters. Filters were probed with amplicons corresponding to either the $f g b A$ (fibrinogen binder A) gene or the deleted portion of the flp1flp $2 f l p 3$ genes. The flp1flp2flp 3 and the fgbA probes were made using primers $\mathrm{P} 9$ and $\mathrm{P} 10$ and primers $\mathrm{P} 11$ and P12, respectively. Probes were labeled with digoxigenin using the DIG DNA Labeling Kit (Roche Applied Sciences, Penzberg, German) and detected with the DIG Easy Hyb protocol (Roche Applied Sciences) according to the manufacturer's instructions.

\section{Adherence assays}

Adherence of bacteria to HFF was measured quantitatively as described previously [4]. Briefly, 24-well tissue culture plates (Costar, Corning, N.Y.) were inoculated with $10^{5} \mathrm{HFF} /$ well and grown to confluence. $35000 \mathrm{HP} \Delta f l p 1-3(\mathrm{pJW} 1), 35000 \mathrm{HP} \Delta f l p 1-3(\mathrm{pLSSK})$, and $35000 \mathrm{HP}$ (pLSSK) were grown in Columbia broth to an $\mathrm{OD}_{660}$ between 0.4 and 0.6 and harvested by centrifugation. Bacterial pellets were suspended using HFF medium and approximately $10^{6} \mathrm{CFU}$ were added to individual wells containing confluent $\mathrm{HFF}$, centrifuged at $500 \times g$, and incubated for $2 \mathrm{~h}$ at $33^{\circ} \mathrm{C}$. After nonadherent bacteria were removed by washing three times with HFF medium, $1 \mathrm{ml}$ of trypsin-EDTA (Invitrogen) solution was added to each well and the plate was incubated for $5 \mathrm{~min}$ to liberate the bound bacteria. Serial dilutions of well contents were plated to quantitate HFF-bound bacteria. Percent adherence was calculated as the ratio of HFF-bound bacteria to initial CFU added per well. A paired Student's t test was used for the comparison of strains' abilities to attach to HFF cells.

\section{Microcolony formation assays}

Confluent HFF monolayers were prepared and 35000HP (pLSSK), 35000HP $\Delta$ flp1-3(pLSSK), and 35000HP $\Delta f l p 1-3$ (pJW1) were grown as described for the adhesion assays. Bacterial pellets were resuspended in HFF medium to an $\mathrm{OD}_{660}$ of 0.1. Approximately $10^{6} \mathrm{CFU}$ for each strain was added to individual wells of confluent HFF cells, centrifuged at $500 \times g$ for $4 \mathrm{~min}$, and incubated for $24 \mathrm{~h}$ at $33^{\circ} \mathrm{C}$. Wells were washed three times with $1 \mathrm{ml}$ HFF medium and then stained with crystal violet $[0.25 \%$ (wt/ vol) crystal violet, $20 \%$ (vol $/ \mathrm{vol})$ methanol, $0.9 \%$ (wt/vol) $\mathrm{NaCl}, 0.02 \mathrm{M}$ Tris- $\mathrm{HCl}(\mathrm{pH} 7.5)]$ for $20 \mathrm{~min}$ at room temperature.

\section{Potential conflicts of Interest}

The authors have no conflicts of interest to report.

\section{Acknowledgements and Funding}

We thank Eric Hansen for sharing antibodies used in this work, and S.M. Spinola and M.E. Bauer for their helpful discussions and critical reviews of the manuscript. We thank the volunteers who enrolled in the human 
challenge study. This work was supported by National Institutes of Health $(\mathrm{NIH})$ National Institute of Allergy and Infectious Diseases (NIAID) grant Al074657 to D.M.J. The human challenge trials were supported by NIH NIAID Public Health Service grant U19 Al31494 and by the Indiana Clinical and Translational Sciences Institute and the Indiana Clinical Research Center (UL RR052761)

\section{Author details}

'Department of Medicine, Indiana University, School of Medicine, Indianapolis, IN 46202, USA. '2Department of Microbiology and Immunology, Indiana University, School of Medicine, Indianapolis, IN 46202, USA. ${ }^{3}$ The Research Institute at Nationwide Children's Hospital, The Ohio State University, Columbus, Ohio 43205, USA. ${ }^{4}$ Department of Pediatrics, The Ohio State University, Columbus, Ohio 43205, USA.

\section{Authors' contributions}

SAC carried out the adherence and microcolony formation assays. JW constructed and characterized the complemented mutant. BB and RSM constructed and characterized the flp 1-3 deletion mutant. KRF prepared the bacterial strains used for the human inoculation experiments and participated in the mutant characterization. BWZ and SE prepared the regulatory documents and performed the clinical observations for the human inoculation experiments. BPK performed the statistical analysis. DMJ and RSM participated in the design of the study and drafted the manuscript. All authors read and approved the final manuscript.

Received: 1 June 2011 Accepted: 22 September 2011 Published: 22 September 2011

\section{References}

1. Schreiner HC, Sinatra K, Kaplan JB, Furgang D, Kachlany SC, Planet PJ, Perez BA, Figurski DH, Fine DH: Tight-adherence genes of Actinobacillus actinomycetemcomitans are required for virulence in a rat model. Proc Natl Acad Sci USA 2003, 100:7295-7300.

2. Tomich M, Planet PJ, Figurski DH: The tad locus: postcards from the widespread colonization island. Nat Rev Microbiol 2007, 5:363-375.

3. Kachlany SC, Planet PJ, Bhattacharjee MK, Kollia E, DeSalle R, Fine DH, Figurski DH: Nonspecific adherence by Actinobacillus actinomycetemcomitans requires genes widespread in Bacteria and Archaea. J Bacteriol 2000, 182:6169-6176.

4. Nika JR, Latimer JL, Ward CK, Blick RJ, Wagner NJ, Cope LD, Mahairas GG, Munson J, Hansen EJ: Haemophilus ducreyi requires the flp gene cluster for microcolony formation in vitro. Infect Immun 2002, 70(6):2965-2975.

5. Spinola SM, Fortney KR, Katz BP, Latimer JL, Mock JR, Vakevainen M, Hansen EJ: Haemophilus ducreyi requires an intact flp gene cluster for virulence in humans. Infect Immun 2003, 71:7178-7182.

6. Alfa MJ, Stevens MK, Degagne P, Klesney-Tait J, Radolf JD, Hansen EJ: Use of tissue culture and animal models to identify virulence-associated traits of Haemophilus ducreyi. Infect Immun 1995, 63:1754-1761.

7. Baba T, Ara T, Hasegawa M, Takai Y, Okumura Y, Baba M, Datsenko KA, Tomita M, Wanner BL, Mori H: Construction of Escherichia coli K-12 inframe, single-gene knockout mutants: the Keio collection. Mol Syst Biol 2006, 2:2006 0008

8. Tracy E, Ye F, Baker BD, Munson RS Jr: Construction of non-polar mutants in Haemophilus influenzae using FLP recombinase technology. BMC Molecular Biology 2008, 9:101.

9. Spinola SM, Fortney KR, Baker B, Janowicz DM, Zwickl B, Katz BP, Blick RJ, Munson RS Jr: Activation of the CpxRA system by deletion of $c p x A$ impairs the ability of Haemophilus ducreyi to infect humans. Infect Immun 2010, 78:3898-3904.

10. Janowicz DM, Ofner S, Katz BP, Spinola SM: Experimental infection of human volunteers with Haemophilus ducreyi: 15 years of clinical data and experience. J Infect Dis 2009, 199:1671-1679.

11. Bauer ME, Fortney KR, Harrison A, Janowicz DM, Munson RS Jr, Spinola SM: Identification of Haemophilus ducreyi genes expressed during human infection. Microbiology 2008, 154:1152-1160.

12. Labandeira-Rey M, Brautigam CA, Hansen EJ: Characterization of the CpxRA Regulon in Haemophilus ducreyi. Infect Immun 2010, 78:4779-4791.

13. Labandeira-Rey M, Dodd D, Fortney KR, Zwickl B, Katz BP, Janowicz DM, Spinola SM, Hansen EJ: A Haemophilus ducreyi cpxR deletion mutant is virulent in human volunteers. J Infect Dis 2011, 203:1859-1865.
14. White $C D$, Leduc I, Jeter C, Harris C, Elkins C: Haemophilus ducreyi outer membrane determinants, including DsrA, define two clonal populations. Infect Immun 2005, 73:2387-2399.

15. Post DMB, Munson RS Jr, Baker B, Zhong H, Bozue JA, Gibson BW: Identification of genes involved in the expression of atypical lipooligosaccharide structures from a second class of Haemophilus ducreyi. Infect Immun 2007, 75:113-121.

16. Bauer ME, Goheen MP, Townsend CA, Spinola SM: Haemophilus ducreyi associates with phagocytes, collagen, and fibrin and remains extracellular throughout infection of human volunteers. Infect Immun 2001, 69:2549-2557.

17. Bauer ME, Townsend CA, Ronald AR, Spinola SM: Localization of Haemophilus ducreyi in naturally acquired chancroidal ulcers. Microbe Infect 2006, 8:2465-2468.

18. Fuller TE, Kennedy MJ, Lowery DE: Identification of Pasteurella multocida virulence genes in a septicemic mouse model using signature-tagged mutagenesis. Microb Pathog 2000, 29:25-38.

19. Harper M, Boyce JD, Wilkie IW, Adler B: Signature-tagged mutagenesis of Pasteurella multocida identifies mutants displaying differential virulence characteristics in mice and chickens. Infect Immun 2003, 71:5440-5446.

20. Kachlany SC, Planet PJ, DeSalle R, Fine DH, Figurski DH, Kaplan JB: flp-1, the first representative of a new pilin gene subfamily, is required for nonspecific adherence of Actinobacillus actinomycetemcomitans. Mol Microbiol 2001, 40:542-554.

21. Labandeira-Rey M, Janowicz DM, Blick RJ, Fortney KR, Zwickl B, Katz BP, Spinola SM, Hansen EJ: Inactivation of the Haemophilus ducreyi luxS gene affects the virulence of this pathogen in human subjects. $J$ Infect Dis 2009, 200:409-416.

22. Al-Tawfiq JA, Thornton AC, Katz BP, Fortney KR, Todd KD, Hood AF, Spinola SM: Standardization of the experimental model of Haemophilus ducreyi infection in human subjects. J Infect Dis 1998, 178:1684-1687.

23. Janowicz DM, Fortney KR, Katz BP, Latimer JL, Deng K, Hansen EJ, Spinola SM: Expression of the LspA1 and LspA2 proteins by Haemophilus ducreyi is required for virulence in human volunteers. Infect Immun 2004, 72:4528-4533.

24. Fulcher RA, Cole LE, Janowicz DM, Toffer KL, Fortney KR, Katz BP, Orndorff PE, Spinola SM, Kawula TH: Expression of Haemophilus ducreyi collagen binding outer membrane protein $\mathrm{NcaA}$ is required for virulence in swine and human challenge models of chancroid. Infect Immun 2006, 74:2651-2658.

25. Fortney KR, Young RS, Bauer ME, Katz BP, Hood AF, Munson RS Jr, Spinola SM: Expression of peptidoglycan-associated lipoprotein is required for virulence in the human model of Haemophilus ducreyi infection. Infect Immun 2000, 68:6441-6448.

26. Wood GE, Dutro SM, Totten PA: Target cell range of Haemophilus ducreyi hemolysin and its involvement in invasion of human epithelial cells. Infect Immun 1999, 67:3740-3749.

27. Spinola SM, Griffiths GE, Bogdan JA, Menegus MA: Characterization of an 18,000 molecular-weight outer membrane protein of Haemophilus ducreyi that contains a conserved surface-exposed epitope. Infect Immun 1992, 60:385-391.

28. Spinola SM, Bong CTH, Faber AL, Fortney KR, Bennett SL, Townsend CA, Zwickl BE, Billings SD, Humphreys TL, Bauer ME, et al: Differences in host susceptibility to disease progression in the human challenge model of Haemophilus ducreyi infection. Infect Immun 2003, 71:6658-6663.

29. Banks KE, Fortney KR, Baker B, Billings SD, Katz BP, Munson RS Jr, Spinola SM: The enterobacterial common antigen-like gene cluster of Haemophilus ducreyi contributes to virulence in humans. J Infect Dis 2008, 197:1531-1536.

\section{doi:10.1186/1471-2180-11-208}

Cite this article as: Janowicz et al.: Expression of the flp proteins by Haemophilus ducreyi is necessary for virulence in human volunteers. BMC Microbiology 2011 11:208. 